Received: 2018.11.01

Accepted: 2018.11.20

Published: 2019.03.16
Authors' Contribution:

Study Design A

Data Collection B

Statistical Analysis C

Data Interpretation D

Manuscript Preparation E

Literature Search F

Funds Collection G

\section{Mukonal Inhibits Cell Proliferation, Alters Mitochondrial Membrane Potential and Induces Apoptosis and Autophagy in Human CNE1 Nasopharyngeal Carcinoma Cells}

\author{
ABCDEF Yingyuan Guo \\ BCDE Yanru Hao \\ ABCDEFG Guofang Guan \\ BCDE Shuaishuai Ma \\ BCDE Zhiling Zhu \\ BCDE Fang Guo \\ BCDE Jie Bai
}

Department of Otolaryngology, The Second Hospital of Jilin University, Changchun, Jilin, P.R. China
Corresponding Author: Source of support:

Background: Nasopharyngeal carcinoma results in high patient morbidity and mortality, due to early metastasis, and toxicity due to chemotherapy. Mukonal is plant-derived carbazole alkaloid that has been used in traditional Chinese medicine to treat several types of cancer. This study aimed to investigate the effects of mukonal on cell proliferation, apoptosis, autophagy, and the mitochondrial membrane potential of nasopharyngeal carcinoma cells in vitro.

Material/Methods: CNE1 human nasopharyngeal carcinoma cells and NP69 normal nasopharyngeal epithelial cells were cultured with and without treatment with increasing doses of mukonal. Cell viability was determined by the MTT assay. Fluorescence microscopy was used to detect reactive oxygen species (ROS), mitochondrial membrane potential, and the release of cytochrome $\mathrm{C}$. Flow cytometry was used to examine changes in the cell cycle, electron microscopy examined cell autophagy, and Western blot was performed to measure levels of proteins associated with autophagy and apoptosis.

Results: $\quad$ Mukonal had an antiproliferative effect on CNE1 cells, with an $\mathrm{IC}_{50}$ of $9 \mu \mathrm{M}$ and there were effects of toxicity on normal NP69 cells. Mukonal triggered ROS-mediated changes in mitochondrial membrane potential which was also accompanied by the discharge of cytochrome C in the CNE1 cells. Mukonal activated autophagy and apoptosis in CNE1 cells, which was also associated with upregulation of the autophagy-related proteins, LC3 II and beclin-1, as well as apoptosis-associated proteins, Bax, cleaved caspase- 3 and -9 . Mukonal treatment also resulted in CNE1 cells cycle arrest at $\mathrm{G}_{2} / \mathrm{M}$.

Conclusions: Mukonal inhibited the growth of human CNE1 nasopharyngeal carcinoma cells in vitro.

MeSH Keywords: Apoptosis • Autophagy • Nasopharyngeal Neoplasms

Full-text PDF: https://www.medscimonit.com/abstract/index/idArt/913915

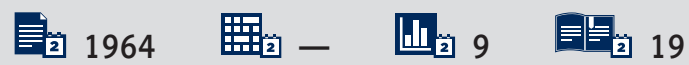




\section{Background}

Nasopharyngeal carcinoma is one of the most prevalent malignant tumors in Southeast Asia and Southern China [1]. This malignancy is associated with high patient morbidity and mortality due to due to early metastasis, and toxicity due to current chemotherapy [1]. The five-year survival rate, using combined treatment with adjuvant cisplatin chemotherapy and radiotherapy, is between $50-60 \%$ [2]. The constant relapses and distant metastasis of nasopharyngeal carcinoma make it difficult to manage with current treatment strategies [3].

Current treatment for nasopharyngeal carcinoma includes surgical excision, systemic chemotherapy, or local radiotherapy. However, owing to the adverse effects of some treatments, patient quality of life is can be impaired [4]. Drug treatment derived from natural sources have recently been studied, and have included studies to identify and screen anticancer molecules from natural resources [5]. Also, the advent of in silico technology has recently helped to identify natural bioactive molecules from the vast libraries of natural products [6]. Based on the structure of their metabolites, herbal-derived therapeutic agents have been classified, and carbazole alkaloids have recently received attention due to their pharmacological potential [7].

Carbazole alkaloids have been shown to have anticancer effects [8]. Mukonal, a carbazole alkaloid derived from Clausena guillauminii has recently been shown to have anticancer effects in different types of cancer [9]. Mukonal has been reported to induce apoptosis and cell cycle arrest of laryngeal cancer cells [9]. Therefore, this study aimed to investigate the effects of mukonal on cell proliferation, apoptosis, autophagy, and the mitochondrial membrane potential of CNE1 nasopharyngeal carcinoma cells in vitro.

\section{Material and Methods}

\section{Reagents, cell lines, and cell culture}

Mukonal, which was determined to be $98 \%$ pure by high-performance liquid chromatography (HPLC), and other chemicals were obtained from Sigma-Aldrich (Sigma-Aldrich, St. Louis MO, USA), unless otherwise stated. The human nasopharyngeal carcinoma cell line, CNE1 and the normal NP69 cell lines were obtained from the Type Culture Collection of Chinese Academy of Sciences (Shanghai, China). RPMI-1640 cell culture media was used, which also contained fetal bovine serum (FBS) (Thermo Fisher Scientific Inc., Waltham, MA, USA) (10\%) and appropriate antibiotics (streptomycin $100 \mu \mathrm{g} / \mathrm{ml}$ and penicillin $\mathrm{G} 100 \mathrm{U} / \mathrm{ml}$ ). The cultures were maintained in an atmosphere containing $5 \% \mathrm{CO}_{2}$ at $37^{\circ} \mathrm{C}$.

\section{Cell viability assay}

Briefly, at around 70\% confluence the CNE1 and the NP69 cells were seeded in 96-well plates and treated with 0-320 $\mu \mathrm{M}$ of mukonal. After incubation for $24 \mathrm{~h}$, the cells were incubated with MTT for $4 \mathrm{~h}$. Then, the media was removed and the colored formazan product was solubilized by $200 \mu$ l of dimethyl sulfoxide (DMSO). The viability of the CNE1 and the NP69 cells was then determined by absorbance measurements at $570 \mathrm{~nm}$.

\section{Determination of reactive oxygen species (ROS), mitochondrial membrane potential, and cytochrome C}

The ROS and mitochondrial membrane potential levels were estimated by culturing the CNE1 cells for 24 at $37^{\circ} \mathrm{C}$, followed by treatment with increasing doses of mukonal $(0,4.5,9$, and $18 \mu \mathrm{M})$ for $24 \mathrm{~h}$. The media was decanted and the cells were treated with $5 \mu \mathrm{M}$ 2',7'-dichlorofluorescein diacetate (DCH-DA) for estimation of ROS, or rhodamine 123 (Rh123) for estimation of mitochondrial membrane potential, by examination of the cells using laser scanning confocal microscopy. Cytochrome $C$ levels were investigated by immunofluorescence microscopy, as previously described [10].

\section{Fluorescence nuclear staining with 4',6-diamidino-2- phenylindole (DAPI)}

CNE1 cells were grown in 6 -well plates $\left(0.6 \times 10^{6}\right.$ cells/well) and incubated for $12 \mathrm{~h}$. CNE1 cells were treated with increasing doses of mukonal $(0,4.5,9$, and $18 \mu \mathrm{M})$ for $24 \mathrm{~h}$ at $37^{\circ} \mathrm{C}$. As the cells became non-adherent, $25 \mu \mathrm{l}$ of cell cultures were placed onto glass slides and stained with DAPI. The slides were then covered with a coverslip and examined with a fluorescence microscope.

\section{Transmission electron microscopy (TEM)}

For electron microscopy, the cells were fixed in the solution of $4 \%$ glutaraldehyde $0.05 \mathrm{M}$ sodium cacodylate, post-fixed in $1.5 \%$ osmium tetroxide $\left(\mathrm{OsO}_{4}\right)$, and dehydrated in alcohol. The cells were then prepared for embedding in Epon 812, sectioned, and then observed using a Zeiss CEM 902 electron microscope (Zeiss, Oberkochen, Germany).

\section{Cell cycle analysis}

After incubating the CNE1 human nasopharyngeal carcinoma cells with increasing concentrations of mukonal $(0,4.5,9$, and $18 \mu \mathrm{M})$ for $24 \mathrm{~h}$. The cells were washed with phosphate buffered saline (PBS). The cells were stained with propidium iodide (PI) and the distribution of the cells in the phases of the cell cycle phases was assessed by fluorescence-activated cell sorting (FACS) flow cytometry. 
A

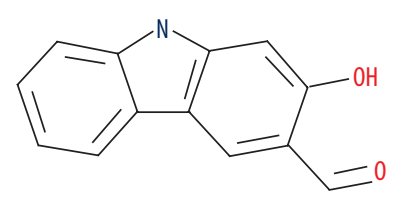

B

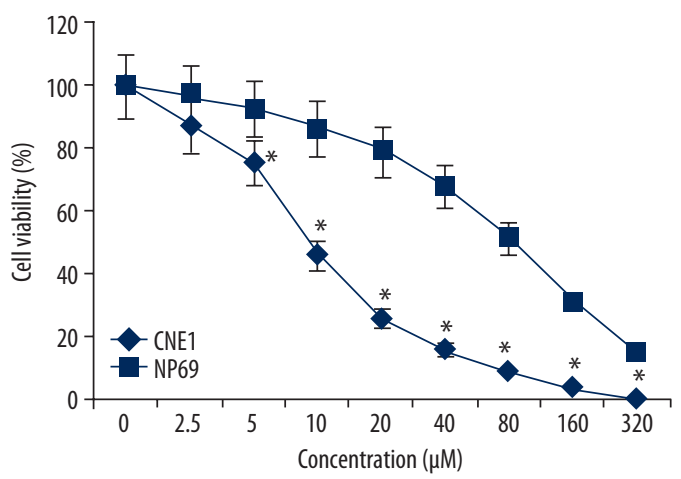

Figure 1. The chemical structure of mukonal and the MTT assay for viability and proliferation of the CNE1 nasopharyngeal carcinoma cells and normal NP69 cells. (A) The chemical structure of mukonal. (B) The MTT assay shows the effect of mukonal on the viability of CNE1 nasopharyngeal carcinoma cells and normal NP69 cells. The experiments were performed in triplicate. The results are shown as the mean $\pm S D\left({ }^{*} p<0.05\right)$.

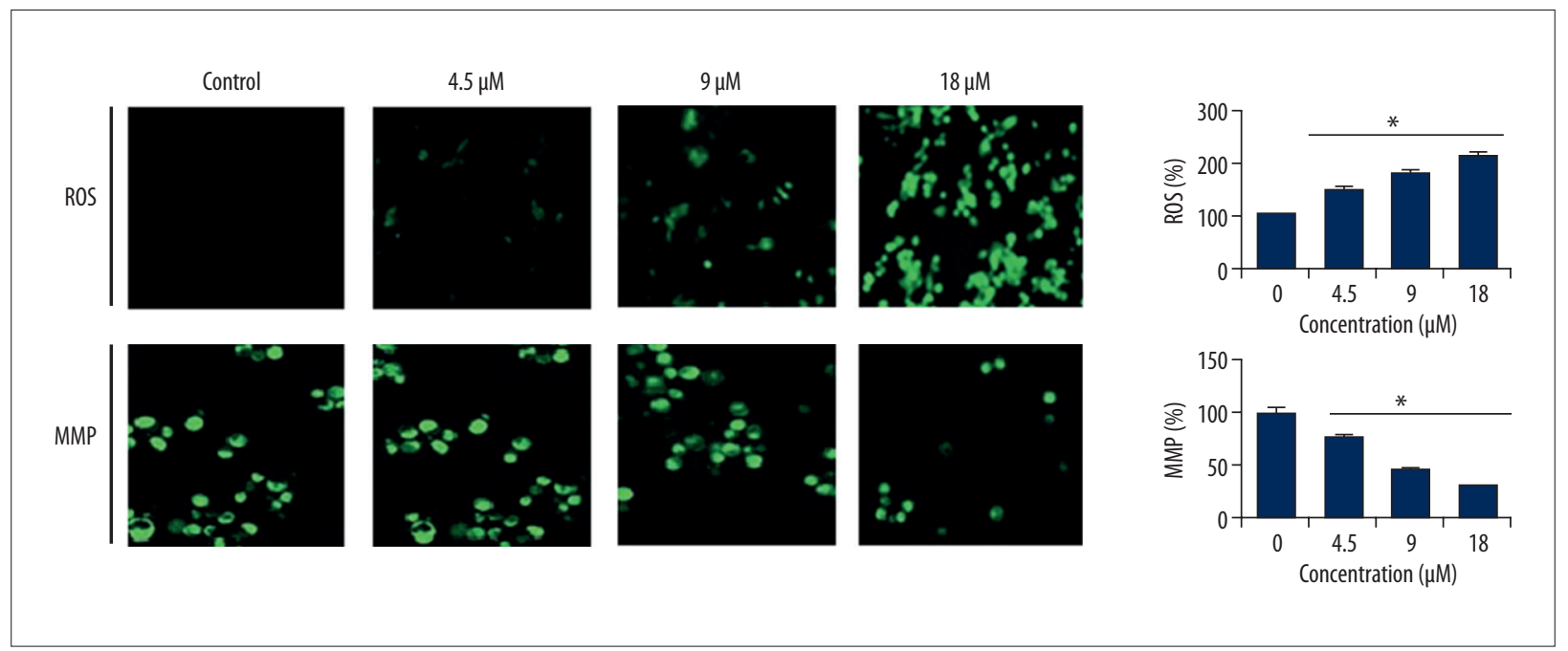

Figure 2. Fluorescence microscopy of mukonal-treated CNE1 nasopharyngeal carcinoma cells shows an increase in reactive oxygen species (ROS) and a decrease in the mitochondrial membrane potential. The experiments were performed in triplicate.

\section{Western blot}

The CNE1 cells were harvested and washed with ice-cold PBS. The cell pellet was then resuspended in a lysis buffer at $4^{\circ} \mathrm{C}$ and then at $95^{\circ} \mathrm{C}$. The protein content of each cell extract was measured using the Bradford spectroscopic assay. About, $40 \mu \mathrm{g}$ of protein was loaded from each sample and separated by sodium dodecyl sulfate-polyacrylamide gel electrophoresis (SDS-PAGE) before being transferred to a polyvinylidene fluoride (PVDF) membrane. The membranes were then washed with TBS and then incubated at $4^{\circ} \mathrm{C}$ in primary antibodies to caspase-3, caspase-9, Bax, Bcl-2, PCNA, cell division cycle $25 \mathrm{C}$ (CDC25C), pCDC25C, CDC2, pCDC2, and cyclin B1. The membranes were incubated with appropriate secondary antibodies and the proteins of interest were visualized by enhanced chemiluminescence (ECL).

\section{Statistical analysis}

Data were presented as the mean \pm standard deviation (SD). Statistical significance and $\mathrm{IC}_{50}$ values were analyzed using GraphPad Prism Demo, Version 5 (GraphPad Software, Inc., San Diego, CA, USA). Student's t-test was used for comparison between two samples, and one-way analysis of variance (ANOVA) followed by Tukey's test was used for comparisons between more than two samples. A P-value $<0.05$ was considered to be statistically significant. 


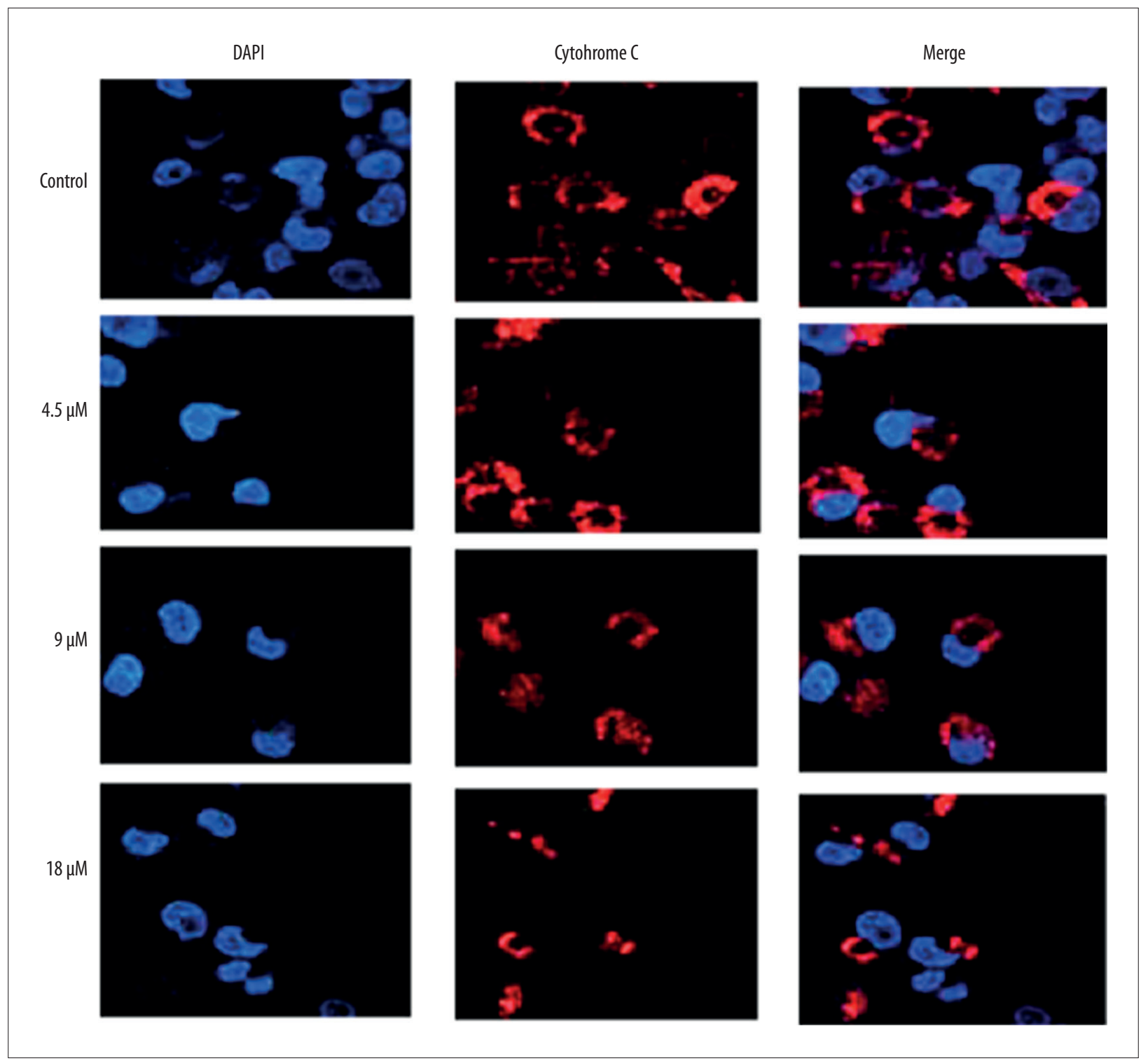

Figure 3. Fluorescence microscopy shows release of cytochrome C from the mukonal-treated CNE1 nasopharyngeal carcinoma cells. The experiments were performed in triplicate.

\section{Results}

\section{Mukonal inhibited the proliferation of CNE1 nasopharyngeal carcinoma cells}

The growth inhibitory effects of mukonal (Figure 1A) were examined on the CNE1 nasopharyngeal carcinoma and the normal NP69 cells by the MTT assay at concentrations ranging from 0 to $320 \mu \mathrm{M}$. Mukonal was found to inhibit the growth of the CNE1 cells in a dose-dependent way (Figure $1 \mathrm{~B}$ ). The $\mathrm{IC}_{50}$ of mukonal for the CNE1 cells was found to $9 \mu \mathrm{M}$. However, the effects of mukonal on the proliferation of the NP69 cells was negligible. The $\mathrm{IC}_{50}$ of mukonal on the normal NP69 cells was $80 \mu \mathrm{M}$ (Figure 1B).
Mukonal induced apoptotic cell death of CNE1 nasopharyngeal carcinoma cells via reactive oxygen species (ROS)-mediated mitochondrial disruption

The levels of ROS and the mitochondrial membrane potential were measured in the CNE1 nasopharyngeal carcinoma cells, and mukonal treatment resulted in the production of large amounts of ROS, and also resulted in the disruption of the mitochondrial membrane potential (Figure 2). Mukonal treatment resulted in the release of cytochrome $C$ in the NCE1 cells (Figure 3). Apoptosis in the mukonal-treated CNE1 cells was determined by fluorescence staining using DAPI, which showed that the percentage of apoptotic cells increased with increasing concentrations of mukonal (Figure 4). Apoptosis of 


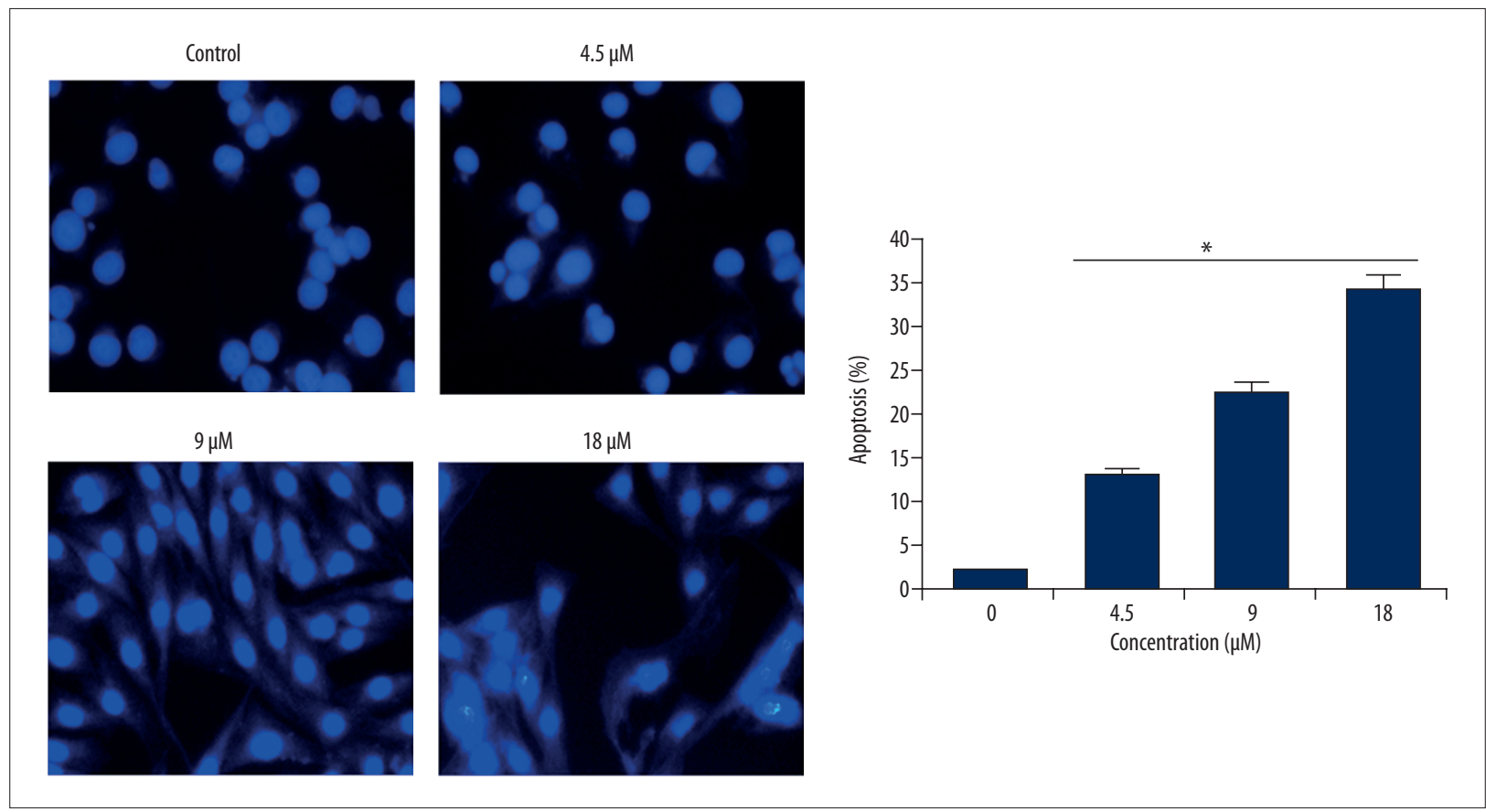

Figure 4. Fluorescence microscopy shows apoptosis of the mukonal-treated CNE1 nasopharyngeal carcinoma cells. Fluorescence staining with 4',6-diamidino-2-phenylindole (DAPI) of the cell nuclei. The experiments were performed in triplicate.

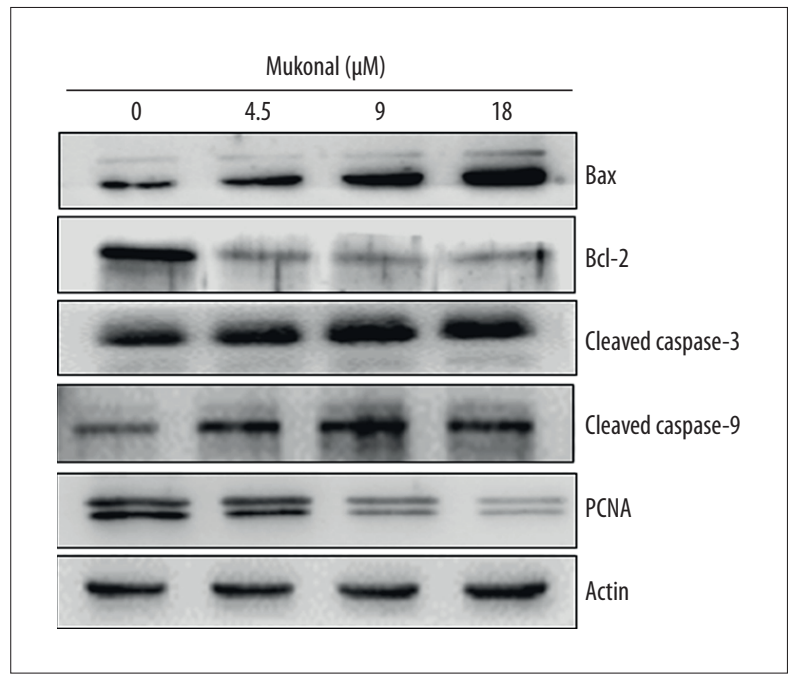

Figure 5. Western blot shows the effect of mukonal treatment on the expression apoptosis-associated proteins in CNE1 nasopharyngeal carcinoma cells. The experiments were performed in triplicate.

the mukonal-treated CNE1 cells was further validated by examining the levels of apoptosis-related proteins by Western blot analysis. Mukonal treatment increased the levels of cleaved caspase- 3 and caspase- 9 in a dose-dependent manner. The expression of Bax was increased, and the expression of $\mathrm{Bcl}-2$ and PCNA were decreased following mukonal treatment (Figure 5).

\section{Mukonal induced autophagy in CNE1 cells}

Electron microscopy was used to further investigate the effects of mukonal on autophagy in the CNE1 cells, and the findings showed that mukonal treatment resulted in the formation of autophagic vesicles in the CNE1 nasopharyngeal carcinoma cells, and this occurred in a dose-dependent manner (Figure 6). Also, mukonal treatment enhanced the expression of LC3 II and beclin-1 and decreased the expression of p62, supporting mukonal-induced autophagy (Figure 7).

\section{Mukonal arrested the CNE1 cells at the G2/M phase of the cell cycle}

The distribution of the cycle phase of CNE1 cells was assessed by flow cytometry at concentrations of $0,9,18$, and $36 \mu \mathrm{M}$ of mukonal. The percentage of cells in the $\mathrm{G} 2 / \mathrm{M}$ phase increased significantly from $6.6 \%$ in the untreated cells to $34.0 \%$ at a concentration of $36 \mu \mathrm{M}$ of mukonal (Figure 8). The arrest of the CNE1 cells at the G2/M phase was associated with changes in the expression of several of the cell cycle-associated proteins. The protein expression of CDC25C, pCDC25C, CDC2, pCDC2, and cyclin B1 were decreased following mukonal treatment (Figure 9). 


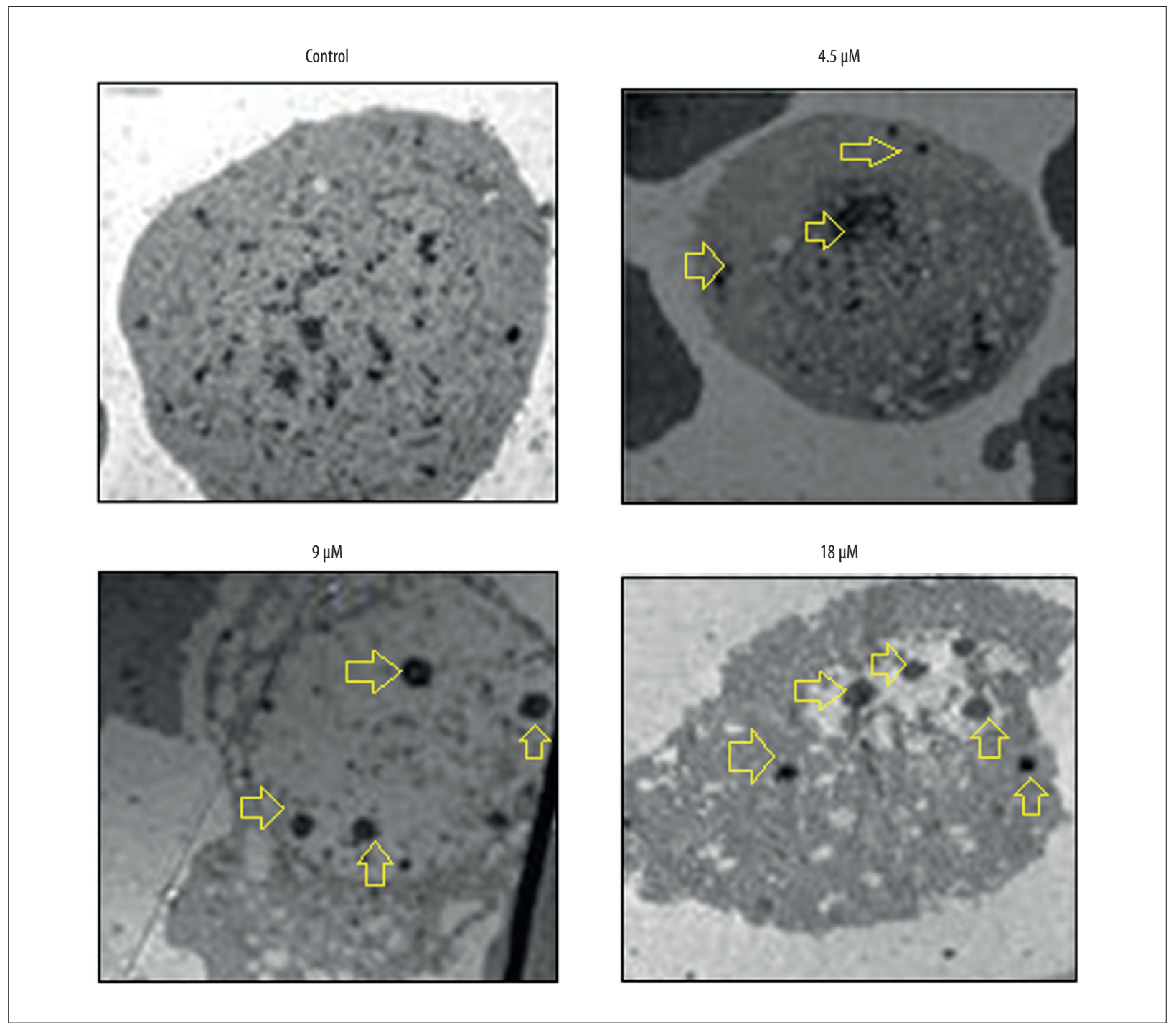

Figure 6. Electron microscopy shows the formation of autophagosomes in the CNE1 nasopharyngeal carcinoma cells. Arrows indicate the autophagosomes. The experiments were performed in triplicate.

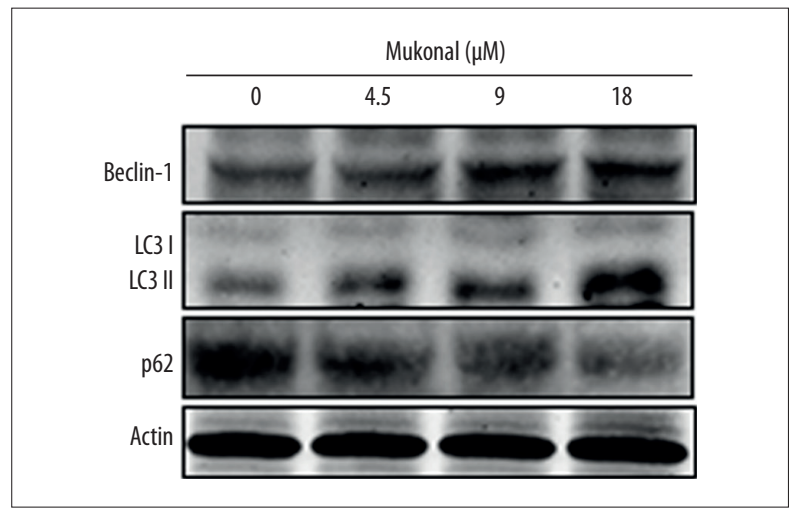

Figure 7. The effect of mukonal on the expression of autophagyassociated proteins in the CNE1 nasopharyngeal carcinoma cells shown by Western blot. The experiments were performed in triplicate.

\section{Discussion}

Nasopharyngeal carcinoma is a common head and neck malignancy [11]. The early metastasis of nasopharyngeal carcinoma and the adverse effects of current treatment strategies are the main obstacles that limit the effectiveness of treatment [12]. Plants serve as a diverse repository of chemicals that may prove to be essential for the development of safer chemotherapy for the treatment of nasopharyngeal carcinoma [13].

In this study, the anticancer effects of mukonal, a plant-derived carbazole alkaloid, were studied in CNE1 human nasopharyngeal carcinoma cells and NP69 normal nasopharyngeal epithelial cells. Mukonal treatment resulted in dose-dependent effects on the CNE1 cells, with minimal toxic effects. These results are in agreement with previous studies that have shown 


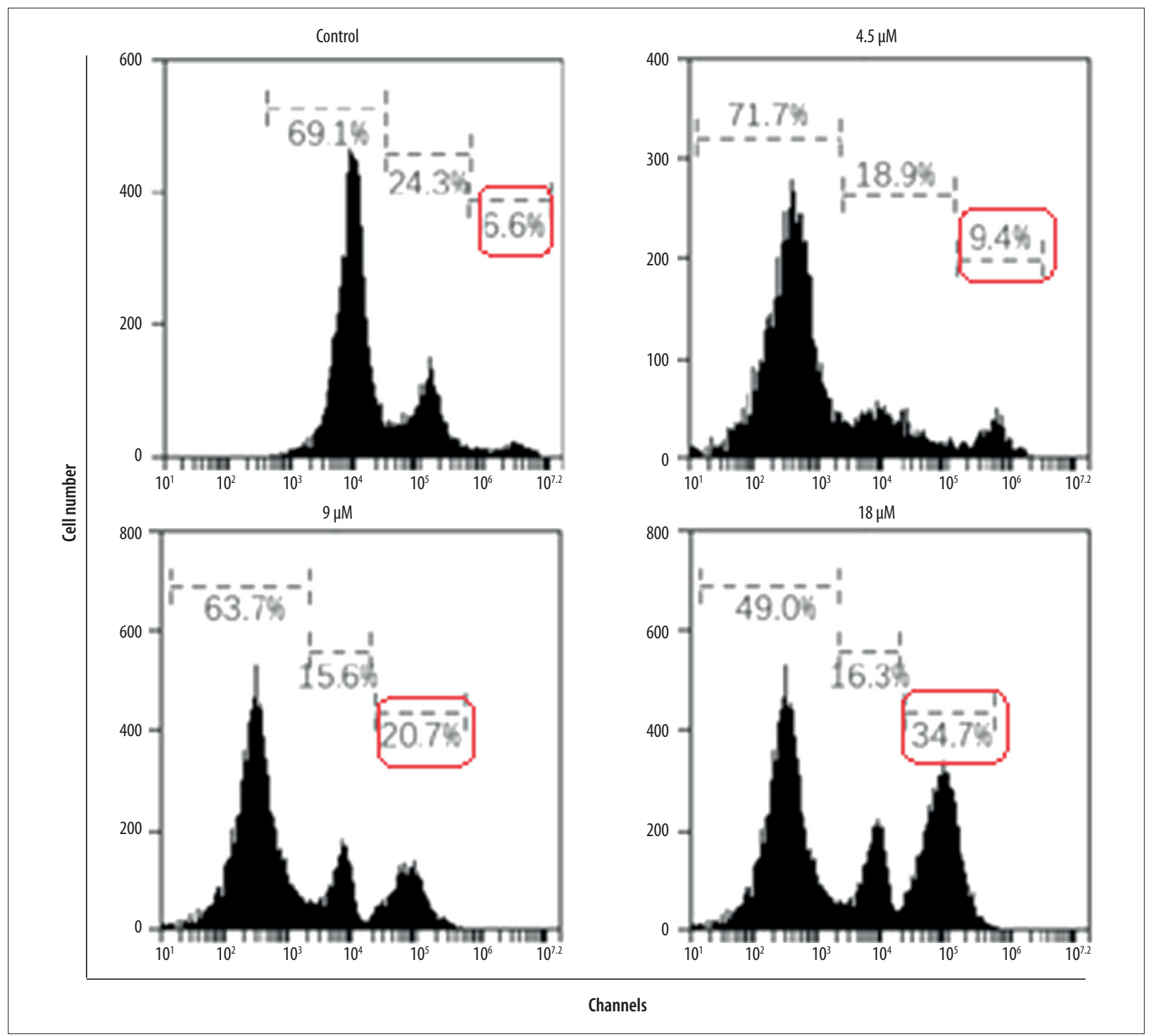

Figure 8. Flow cytometry shows the effects of mukonal on the distribution of the CNE1 nasopharyngeal carcinoma cells in different phases of the cell cycle. The experiments were performed in triplicate.

that alkaloids inhibit the growth of cancer cells [14]. Also, mukonal has previously been shown to inhibit the growth of laryngeal cancer cells, which is a finding that supports the results of this study [9]. The results of this study showed that mukonal caused the accumulation of significant amounts of reactive oxygen species (ROS) that resulted in the disruption of the mitochondrial membrane potential and triggered the release of cytochrome C in CNE1 nasopharyngeal carcinoma cells. Mukonal treatment was associated with apoptosis, which was shown by fluorescence staining of the cell nuclei with 4',6-diamidino2-phenylindole (DAPI), as well as the expression of apoptosisassociated proteins in CNE1 cells. Also, mukonal treatment induced autophagy of the nasopharyngeal carcinoma CNE1 cells and triggered the G2/M arrest in a dose-dependent manner.
Plant-derived molecules have been reported to affect ROS levels in the cancer cells [15]. In the present study, mukonal was shown to trigger the formation ROS in CNE1 cells, which was accompanied by the disruption of the mitochondrial membrane potential, which has previously been reported to be associated with the release of cytochrome C [16]. The findings of this study also showed that mukonal treatment resulted in the release of cytochrome $\mathrm{C}$ in the CNE1 cells. Because apoptosis is an important mechanism in the control of cancer growth and in the maintenance of tissue homeostasis, and mukonal has been shown to be associated with apoptotic cell death in the laryngeal carcinoma cells $[9,17]$, this study included DAPI staining of the mukonal treated-CNE1 cells, which confirmed the effect of mukonal on apoptosis. 


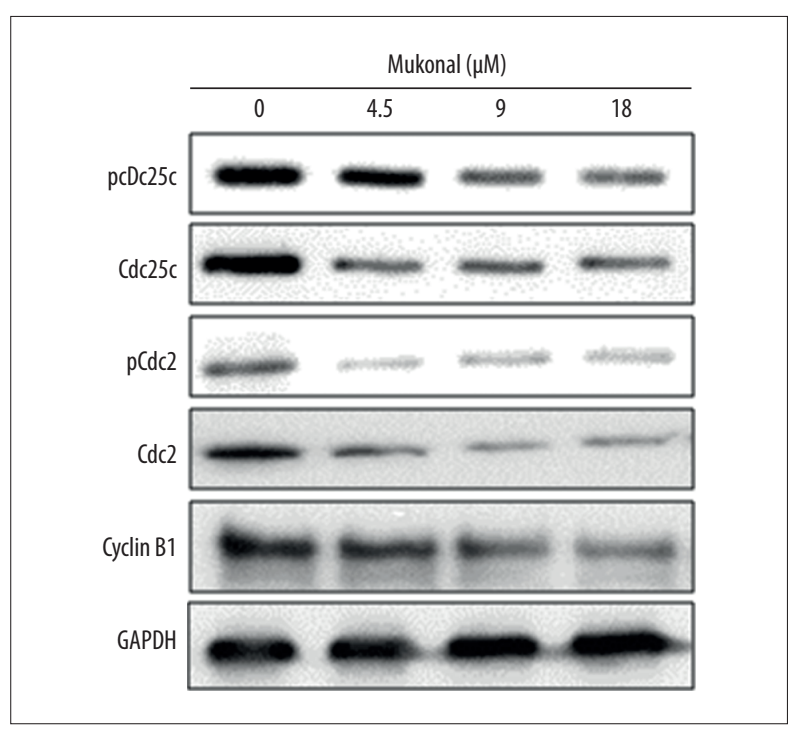

Figure 9. Western blot shows the effect of mukonal on the expression cell cycle-associated proteins. The experiments were performed in triplicate.

In this study, mukonal-induced apoptosis was accompanied by an increase in caspases and Bax and a decrease in Bcl-2 and PCNA. Previous studies have shown that carbazole alkaloids have been shown to induce autophagy in cancer cells. Isomahanine, a carbazole alkaloid, has been reported to activate

\section{References:}

1. Xiao X, Zhang Z, Chang ET et al: Medical history, medication use, and risk of nasopharyngeal carcinoma. Am J Epidemiol, 2018; 26: 2117-25

2. Adham M, Kurniawan AN, Muhtadi Al et al: Nasopharyngeal carcinoma in Indonesia: Epidemiology, incidence, signs, and symptoms at presentation. Chinese J Cancer, 2012; 31(4): 185-96

3. Chang ET, Adami HO: The enigmatic epidemiology of nasopharyngeal carcinoma. Cancer Epidemiol Biomarkers Prev, 2006; 15(10): 1765-77

4. Haleshappa RA, Thanky AH, Kuntegowdanahalli L et al: Epidemiology and outcomes of nasopharyngeal carcinoma: Experience from a regional cancer center in Southern India. South Asian J Cancer, 2017; 6: 122-24

5. Newman DJ, Cragg GM: Natural products as sources of new drugs from 1981 to 2014. J Nat Prod, 2016; 79: 629-61

6. Harvey AL, Edrada-Ebel R, Quinn RJ: The re-emergence of natural products for drug discovery in the genomics era. Nat Rev Drug Discov, 2015; 14(2) $111-29$

7. Fu YH, Ma YL, Yan G et al: Carbazole alkaloids from Clausena emarginata with their potential antiproliferative activities. Nat Prod Res, 2018 [Epub ahead of print]

8. Samanta SK, Kandimalla R, Gogoi B et al: Phytochemical portfolio and anticancer activity of Murraya koenigii and its primary active component, mahanine. Pharmacol Res, 2017; 23: 227-36

9. Li L, Huizhi L, Binu W et al: Anticancer activity of mukonal against human laryngeal cancer cells involves apoptosis, cell cycle arrest, and inhibition of PI3K/AKT and MEK/ERK signalling pathways. Med Sci Monit, 2018; 24 7295-302

10. Chen T, Pengetnze Y, Taylor C: Src inhibition enhances paclitaxel cytotoxicity in ovarian cancer cells by caspase-9-independent activation of caspase-3. Mol Cancer Ther, 2005; 2: 217-24 autophagy in oral carcinoma cells [18]. Therefore, in this study, mukonal-treated CNE1 cells were examined by electron microscopy and resulted in the formation of autophagic vesicles in the CNE1 cells. Also, mukonal treatment upregulated the expression of LC3 II and beclin-1, which are markers of autophagy. Mukonal treatment also arrested CNE1 cells in the $\mathrm{G} 2 / \mathrm{M}$ phase in a dose-dependent manner. These findings are supported by the findings from studies on similar molecules, such as mahanimbine, which has been reported to trigger cell cycle arrest [19]. The results of the present study suggest that further studies, including in vitro and in vivo studies, and controlled clinical studies, are required to evaluate the potential role of mukonal in the management of nasopharyngeal cancer.

\section{Conclusions}

Mukonal inhibited the growth of CNE1 nasopharyngeal carcinoma cells in vitro without toxicity and triggered apoptosis, autophagy, and cell cycle arrest of the CNE1 cells via reactive oxygen species (ROS)-mediated alterations in the mitochondrial membrane potential. These findings support the need for further in vitro and clinical studies to determine the potential role for mukonal as a natural treatment for nasopharyngeal carcinoma.

11. Goh TH, Yip WK, Seow HF: Synergistic combinations of small molecule kinase inhibitors: Implications for reducing toxicities in nasopharyngeal carcinoma treatment. J Nasopharyng Carcinoma, 2017; 4(4): e38

12. Crooker $\mathrm{K}$, Aliani $\mathrm{R}$, Ananth $\mathrm{M}$ et al: A review of promising natural chemopreventive agents for head and neck cancer. Cancer Prev Res, 2018; 11(8): 441-450.

13. Dahiya J, Singh J, Kumar A, et al. Isolation, characterization and quantification of an anxiolytic constituent-mahanimbine, from Murraya koenigii Linn. spring leaves. J Ethnopharmacol, 2016; 193: 706-11

14. Utaipan T, Athipornchai A, Suksamrarn A et al: Carbazole alkaloids from Murraya koenigii trigger apoptosis and autophagic flux inhibition in human oral squamous cell carcinoma cells. J Nat Med, 2017; 71(1): 158-69

15. Hua F, Li CH, Chen XG et al: Daidzein exerts anticancer activity towards SKOV3 human ovarian cancer cells by inducing apoptosis and cell cycle arrest, and inhibiting the Raf/MEK/ERK cascade. Int J Mol Med, 2018; 41(6): 3485-92

16. Dong X, Fu J, Yin X et al: Induction of apoptosis in HepaRG cell line by aloe-emodin through generation of reactive oxygen species and the mitochondrial pathway. Cell Physiol Biochem, 2017; 42(2): 685-96

17. Lopez J, Tait SW: Mitochondrial apoptosis: Killing cancer using the enemy within. Br J Cancer, 2015; 112(6): 957-62

18. Utaipan T, Athipornchai A, Suksamrarn A et al: Isomahanine induces endoplasmic reticulum stress and simultaneously triggers p38 MAPK-mediated apoptosis and autophagy in multidrug-resistant human oral squamous cell carcinoma cells. Oncol Rep, 2017; 37(2): 1243-52

19. Pei C, He Q, Liang S, Gong X: Mahanimbine exerts anticancer effects on human pancreatic cancer cells by triggering cell cycle arrest, apoptosis, and modulation of AKT/mammalian target of rapamycin (mTOR) and signal transducer and activator of transcription 3 (STAT3) signalling pathways. Med Sci Monit, 2018; 24: 6975-83 